\title{
Preliminary Results on Multi Offset GPR for Imaging of Landmines
}

\author{
Federico Lombardi and Hugh D. Griffiths \\ Department of Electrical and \\ Electronic Engineering \\ University College London \\ London, WC1E6BT, United Kingdom \\ f.lombardi@ucl.ac.uk, h.griffiths@ucl.ac.uk
}

\author{
Alessio Balleri \\ Centre for Electronic Warfare, \\ Information and Cyber \\ Cranfield University \\ Defence Academy of the UK \\ Shrivenham, SN68LA, United Kingdom \\ a.balleri@cranfield.ac.uk
}

\author{
Maurizio Lualdi \\ Department of Civil and \\ Environmental Engineering \\ Politecnico di Milano \\ Milan, 20133, Italy \\ maurizio.lualdi@polimi.it
}

\begin{abstract}
Ground Penetrating Radar (GPR) is widely recognised as an operationally useful sensor for mine detection as it can offer better detection performance than the ubiquitous metal detector in the presence of low-metal content mines. However, GPR has to overcome many potential sources of false alarm due to clutter and battlefield debris, which lower the efficiency of the sensor. This paper analyses a set of experimental data collected in a recent multi-offset GPR measurement campaign with inert landmines composed of different assemblies buried in sandy soil. The aim of the work is to evaluate the key differences observed by a radar system when the transmitter and the receiver are moved apart, as a function of their distance and hence when the illuminated section of the target is diversified. The results of the comparison between the collected multi-offset profiles show that using a bistatic geometry could represent a strategy to reconstruct composite objects with finer and better details.
\end{abstract}

\section{INTRODUCTION}

Landmines pose a serious and ongoing threat to civilians, instilling fear in communities and acting as a lethal barrier to development and post-conflict reconstruction [1]. Currently, 11 states are identified as producers of antipersonnel landmines (APL), with many more reserving the right to do so, and more than 60 countries and areas are contaminated by mines as October 2016 [2].

Reliable mine detection is still an unresolved problem. Demining operations are complicated because of the large variety of existing landmine types, many different possible soil and terrain conditions, and environmental circumstances [3]. Traditional approaches to detect landmines use electromagnetic induction (EMI) sensors which were firstly developed during WWI and subsequently refined [4]. The advantage of these sensors is that they can sense as little as less than $1 \mathrm{~g}$ of metal and are easy to operate. However, there are significant limitations to this technology that remain to be addressed. Low and non-metallic landmines, for instance, remain one of the most difficult subsurface targets to be detected not only with EMI technique, but also using several other geophysical techniques [5] [6].

Ground Penetrating radar (GPR) has been proposed as an alternative to classical electromagnetic induction techniques for the landmine detection [7] [8] [9] due to a number of advantages [10] [11]. Firstly, GPR can locate and characterise both metallic and non-metallic subsurface features [12] [13] and therefore be used to detect non-metallic APLs [14]. Secondly, generating an image of the mine or other buried objects based on dielectric constant variations can help classify the detected targets and characterise the surrounding area [15] [16] [17]. Finally, GPR sensors are becoming more and more lightweight, easy to operate, and they can scan at a rate comparable to that of EMI systems [18]. Despite these advantages, GPRs still present some technological limitations such as a prohibitive false alarm rate, especially in heterogeneous soil, and a sometimes limiting long acquisition time [19] [20].

Traditional GPR surveys are conducted in quasi-monostatic configurations, with the transmitter and the receiver close to each other, along a single bidimensional scan line. From a practical point of view, this method allows easy access to most survey areas and a relatively fast and simple acquisition [21]. Although most practical applications have been in the monostatic domain, using bistatic geometries may offer several key benefits, especially for low-observable targets in highly heterogeneous soil conditions. Both object detection and identification can be significantly enhanced through the use of the additional degrees of freedom provided by bistatic geometries [22], dimension which increases the measured information content [23]. The multi-offset method, in which the transmitter and the receiver are independently managed, is a well-rehearsed technique developed by the seismic community with the aim of improving Signal to Noise Ratio (SNR) and the accuracy of velocity estimation [24] [25]. The expected potential of using bistatic solutions is to yield lighter weight, lower cost systems and improved detection, identification and coverage performance.

The majority of landmines are moulded from plastic materials and any metallic content is minimal or absent; this means that a change in the distance between the transmitter and the receiver will vary the vertical position of the reflection centre of the overlapping footprint inside the target. Given the nature of the investigated items, one can hypothesise that multiple looks at a target, resulting by varying the spacing between the Tx and Rx antennas, can ease the task of resolving targets of 
interest from clutter and provide a more detailed image of the subsurface features [26] [27] [28].

Migration algorithms, based either on diffraction compensation or waveform inversion, depend on the distance between antennas, thus a better and finer target reconstruction is expected from the combination of the different output of a multi-offset survey [29]. In this way, all information present in the GPR traces is used, which enables the estimation of quantitative electromagnetic properties.

This work presents some preliminary experimental results obtained from the analysis of landmine GPR profiles collected with different transmitter and receiver separation. Multi-offset data were collected for a representative inert landmine buried in sharp sand soil with a GPR consisting of two separate Tx and $\mathrm{Rx}$ modules both placed in contact with the surface to ensure a proper coupling and increase the radiation efficiency. The aim is to provide an initial evaluation of what can be gained from the analysis of multi-offset GPR images, both in terms of level of information and resolution.

The paper is organised as follows. Section II describes and justifies the experimental setup employed in the experiments. Section III presents and comments the results of the campaign exploiting the information content and the features extracted from the collected bistatic profiles for each transmitter/receiver location. Further considerations, the conclusions and suggested future works are provided in Section IV.

\section{EXPERIMENTAL CAMPAIGN}

The bistatic profiles presented in this paper have been acquired in a test sand pit located at the Defence Academy of the United Kingdom in Shrivenham. The GPR equipment used for the measurements consists of an IDS Aladdin radar and an IDS THRHF radar, both provided by IDS Georadar srl. The two impulsed devices carry dipole antennas with an operating central frequency and a bandwidth of $2 \mathrm{GHz}$ and $3 \mathrm{GHz}$, respectively (Figure 1(a)). A soft pad, the PSG (Pad System for Georadar, U.S. Patent no. US 7,199,748 B2 of Politecnico di Milano, Italy, [30]), was placed between the radar equipment and the soil to ensure accurate measurements and fixed antenna orientation from trace to trace [31] (Figure 1(b)). The higher frequency equipment acted as the receiver module to take advantage of the finer sensitivity of its components.
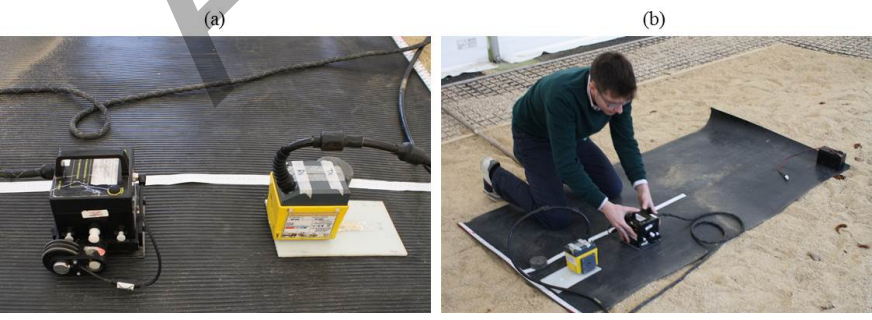

Fig. 1. Data acquisition detail. (a) GPR platforms. Left: $2 \mathrm{GHz}$ antenna; Right: $3 \mathrm{GHz}$ one. (b) Data collection over the surface pad.
Stand-off radar systems suffer from a low energy coupling process efficiency since, in the presence of lossy materials, complex angles of refraction may occur. At larger incident angles than the Brewster angle the losses at the air/ground interface increase rapidly [32]. In addition to the problem of coupling energy into the ground, the effective cross section of all landmines decreases when they are buried. Much of the literature focuses on advanced signal processing techniques in an attempt to remove the effects of the rough surface [33], [34]. The efficacy of conventional air-coupled GPR has been limited by the rough air/ground interface, below which landmines are typically buried. Conversely, employing a ground coupled platform could greatly improve signal penetration and data resolution [35] [36]. When the antennas are in contact with the ground, the subsurface waveform is nearly unaffected by the roughness of the soil and therefore is predictable and easy to analyse, even for plastic landmines [37]. Figure 2 presents an example of the effects originated from elevating the antennas from the ground.
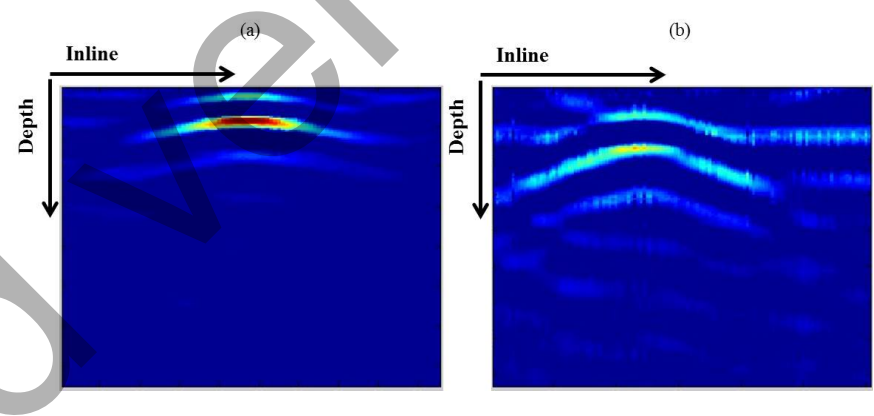

Fig. 2. Example of results from (a) ground coupled and (b) stand-off GPR (antenna height equal to $4 \mathrm{~cm}$ ).

As can be seen, not only the overall quality of Figure 3(a) is lower but also the resolution is degraded. Therefore, when the purpose of the acquisition is not just a detection task, and the aim is to extract details of the internal structure or define the shape of the buried object, increasing the efficiency of the energy spreading may be essential. The major drawbacks of having a device in direct contact with the surface are an accidental detonation of a surface laying device, and the need for a clean terrain surface. Both of them are partially resolved by the necessary prior vegetation-cutting process [38], that for instance occupies about $80 \%$ of the time spent for the clearance process [39], which could create a suitable surface preparation and at the same time eliminate trip wires activated ordnance and surface lying ones. In addition, the GPR platform could be made so light that its weight would not be able to trigger a landmine, and proper armoured surface pads can be employed [40].

The chosen measurement methodology was a common receiver scheme, in which for each receiver position the transmitter antenna was moved along an inline profile (Figure 1(b)). Then, the receiver was progressively shifted along the same profile replicating the previous step. The acquired 
traces were finally arranged to create a set of multi-offset profiles (Figure 3), in which the same GPR scan is defined by a different transmitter and receiver separation. Acquisition details are provided in Table I.
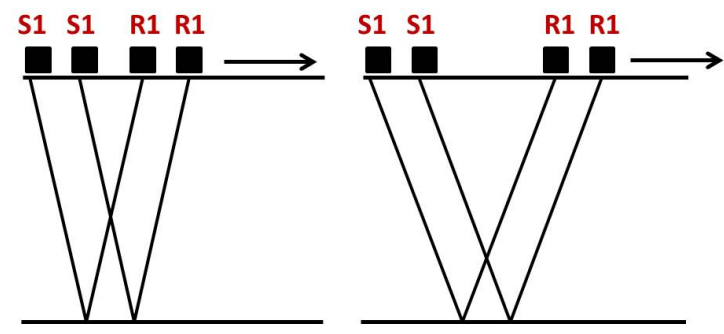

Fig. 3. Data acquisition scheme.

TABLE I

EXPERIMENTAL CAMPAIGN DETAILS

\begin{tabular}{|c|c|}
\hline Parameter & Value \\
\hline Minimum offset & $6 \mathrm{~cm}$ \\
Maximum offset & $40 \mathrm{~cm}$ \\
Offset step & $1 \mathrm{~cm}$ \\
Time window & $30 \mathrm{~ns}$ \\
Time sampling & $0.0587 \mathrm{~ns}$ \\
Target depth & $10 \mathrm{~cm}$ \\
\hline
\end{tabular}

The landmine under test was a Soviet PFM-1 landmine [41] filled with a training simulants for high explosive and complete of all its parts (Figure 4). Although the PFM-1 contains liquid plastic explosive with higher dielectric values, the expected differences in the signature are limited to a variation in magnitude and therefore their effect on the scope of this investigation can be ignored. (a)

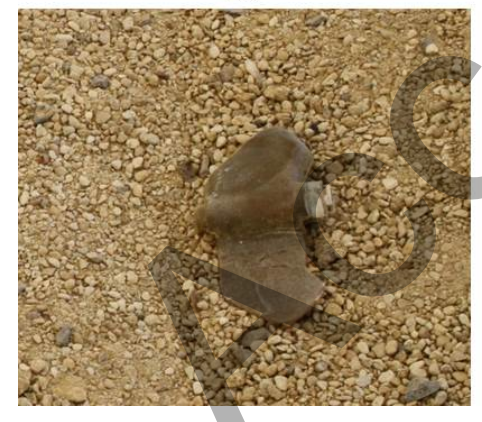

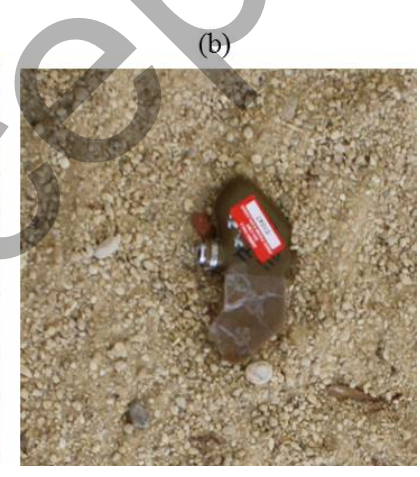

Fig. 4. Employed landmine. (a) Upper side. (b) Lower side.

Figure 5 shows the employed device, together with its detailed components.

The chosen device does not present a complex internal structure but is characterised by a composite shape: a stabiliser wing (marked $\mathrm{C}$ in Figure 5(b)), a detonator cap (marked B in Figure 5(b)), and the main body of the landmine
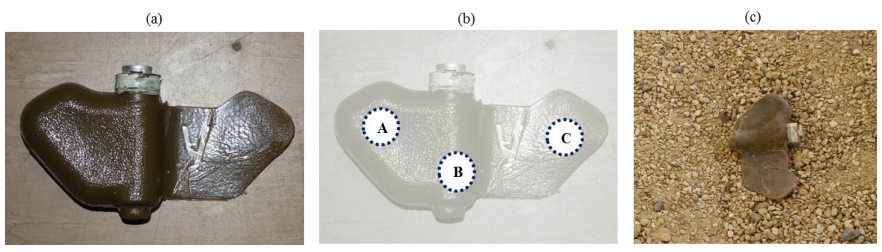

Fig. 5. Soviet PFM-1 details. (a) Representative inert model. (b) Device details. (c) Acquisition geometry.

(marked A in Figure 5(b)). The thickness of the stabiliser wing and the main body is $3 \mathrm{~mm}$ and $20 \mathrm{~mm}$, respectively. The target was buried accordingly to Figure 5(c), the bottom being the deeper limit of the landmine and the stabiliser wing the first encountered components. Data were collected with the reflection centre of the antenna right in the middle of the target.

Due to humidity, the sand was not completely dry, providing a relative dielectric constant of approximately 9 and hence a resulting propagation velocity of $10 \mathrm{~cm} / \mathrm{hs}$. As it can be noticed in Figure 4, the target was buried in sharp sand, with very low clay content and gritty texture for a better drainage. This last aspect was fundamental to avoid trench effects when burying the target in humid conditions.

The processing chain applied to the data [42] consisted of a linear frequency filtering and a spherical exponential compensation gain function, matched to the soil characteristics, to recover the amplitude losses. The particular design of the target should highlight possible variations, as increasing the distance between the transmitter and the receiver will produce an overlapping footprint located at a different depth of the target, as sketched in Figure 6. (a)

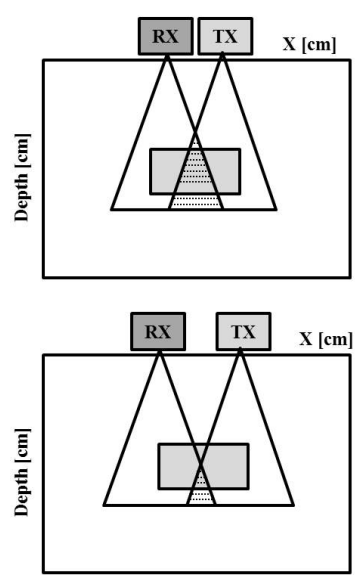

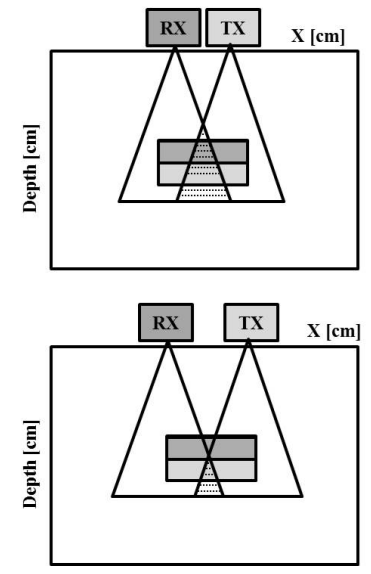

Fig. 6. Sketch of reflection point variations with antennae offset. (a) Solid object. (b) Composite target.

For the case under investigation, a small offset should be capable of fully describing the device, thin wing included, while an increased distance will not manage to image all the landmine details, but it will better define the physical 
extension of the buried target.

\section{RESULTS}

The obtained bistatic profiles are shown in Figure 7. The relative distance between the transmitter and the receiver antenna is indicated at the top of each frame. An interpretative sketch is provided in the lower corner of the figure. In addition, for a proper comparison, all the presented profiles have been normalised.
Offse t: $20 \mathrm{~cm}$

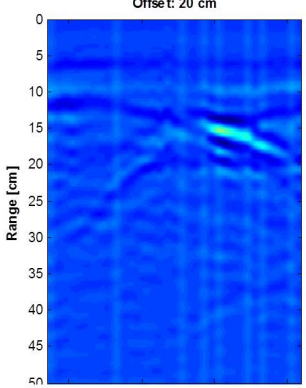

Offset: $35 \mathrm{~cm}$

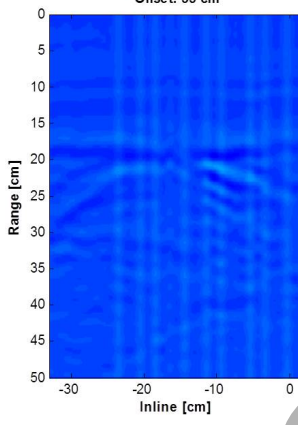

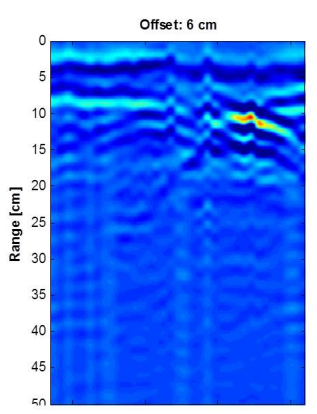

Offset: $10 \mathrm{~cm}$

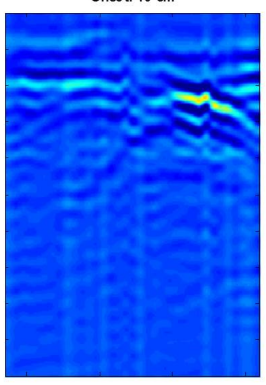

Offset: $25 \mathrm{~cm}$

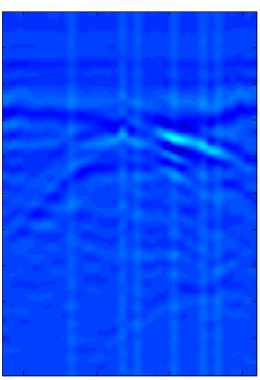

Offset: $40 \mathrm{~cm}$

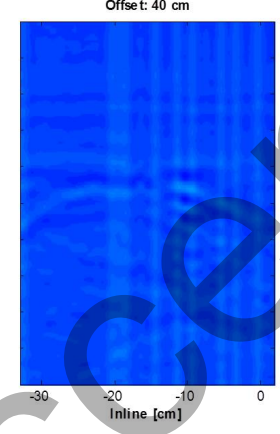

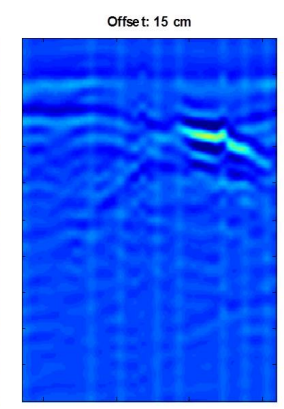

Offset: $30 \mathrm{~cm}$
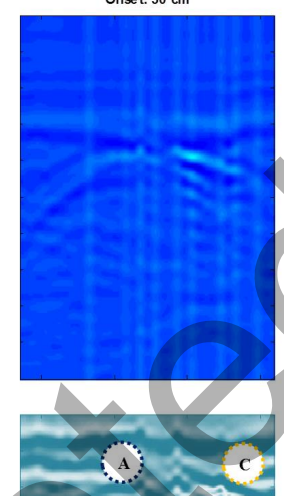

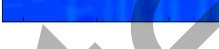

(a)

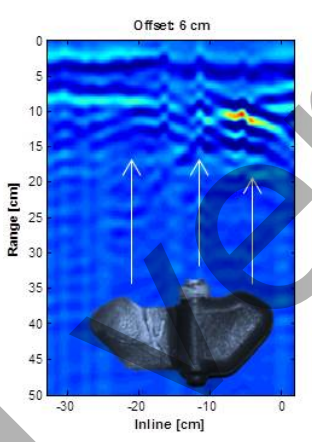

(b)

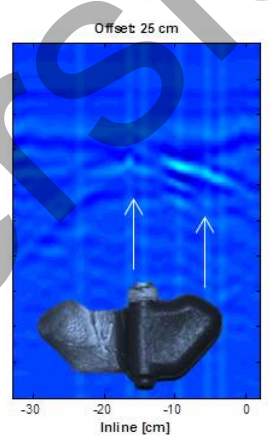

(c)

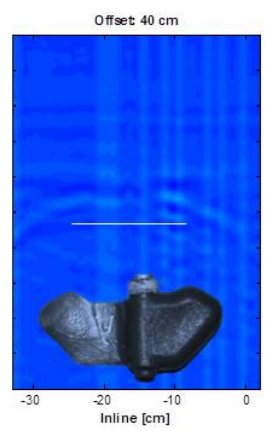

Fig. 8. Interpretative diagram. (a) Wing and main body. (b) Main body. (c) Lower limit.

As a result of its limited thickness, and therefore to its weak scattering magnitude, even a small increase in spacing between antennas can reduce the capability of imaging the stabiliser wing. When the offset is larger than $30 \mathrm{~cm}$, the image delineates the physical extension of the landmine, as the vertical position of the reflection point is located over the lower surface of the target. Figure 8(c) shows a good agreement with the actual size of the landmine, which is approximately $15 \mathrm{~cm}$.

Globally, what can be infer from the frames of Figure 7 is the presence of a target composed of more than a single body, as otherwise the trend of the detected hyperbola would have been constant. Equally, the situation of having two targets located near to each other can be excluded.

As one of the main limitations of GPR as a landmine detector is the common deception of the equipment from clutter sources, through this methodology one can infer with a higher confidence whether the detected anomaly is mainly a solid homogeneous object, hence more likely to be a false alarm, or the presence of a number of assemblies could mark a buried threat.

\section{CONCLUSION}

The reported empirical work has been aimed at investigating the effects of a change in the transmitter and receiver distance on the imaging performance of GPR. The experiments, carried propagation path. For the same reason, the energy content of the frame decreases due to absorption phenomenon as the
The offset range was selected to (1) ensure that all land-
pacing between transmitter and receiver increases. mine reflections were properly collected, and (2) to keep
the maximum spacing corresponding to the actual logistical circumstances. A secondary consideration is the demonstration of the need for high frequency and wide bandwidth systems Comparing the radar response at different offset supports imaging performance, as in a quasi-monostatic case, all the three components highlighted in Figure 5(b) appears in the radargram (Figure 8(a)), while when the footprint illuminates a different section of the target, either the main body only (Figure 8(b)) or its bottom (Figure 8(c)) are visible. 
out with wide bandwidth equipment and with a target buried in sandy soil, have demonstrated that a multi-offset approach can offer a deeper insight of the target, as well as a better understanding of its nature. The level of detail of composite targets can be significantly enhanced comparing the radar response collected at increasing (or decreasing) transmitter and receiver distances.

The radar platform was placed directly over the surface to take advantage of an increased coupling efficiency and avoid the loss of energy at the air/ground interface. A soft pad, potentially moulded with armouring capability, was placed in between to guide the acquisition and to ensure a proper adherence. The employed target, a Soviet PFM-1 landmine was chosen due to its composite design to exploit whether the collected images will show noticeable information content distinction and can be a further tool for reducing the uncertainty in the detection process.

Different behaviours have been highlighted, mainly a significant variation in the information content of a monostatic image compared to the one produced by a large offset. These behaviours are due to the fact that the reflection point will be located on a different part of the target, illuminating different sections. A further valuable consideration could include a combination scheme of the collected profiles to take advantage of the multi-fold properties and increase the information richness of the images.

Obviously, the propagation characteristics of the soil were highly favourable, thus a trial with more heterogeneous soil need to be carried out in order to assess the performance of the technique when the target is buried in lossy ground. Additional future work includes an evaluation of the possibility of combining multi-offset images into a single bidimensional profile with improved quality, with a dedicated processing development and inversion techniques which could take into account the separation variability.

\section{ACKNOWLEDGMENT}

The authors thank the Find A Better Way charity for their support of this research under the DETERMINE programme (grant number 2015/001D). We also thank the Defence Academy Ammunition Hall for providing the representative landmines model used for the experiments and Dr. Francesco Fioranelli for assisting during the experimental campaign. We thank IDS Georadar srl for the provision of the GPR equipment.

\section{REFERENCES}

[1] International Campaign to Ban Landmine, http://www.icbl.org/.

[2] Landmine and Cluster Munition Monitor, http://www.the-monitor.org/.

[3] Bruschini, C., and Bertrand, G. A survey of research on sensor technology for landmine detection. Journal of Conventional Weapons Destruction, 2, 1, 2016.

[4] Sahli, H., Bruschini, C., and Crabbe, S. Catalogue of advanced technologies and systems for humanitarian demining, EUDEM2 Technology Survey, 1, 3, 2005.

[5] Houser, L., Assessing Landmine Detection Using Geophysical Equipment in Sand and Soil Beds Similar to Donetsk Region of Eastern Ukraine, Doctoral Dissertation, 2016.
[6] McDonald, J., Lockwood, J.R., McFee, J., Altshuler, T., Broach, T., Alternatives for landmine detection, DTIC Document, 2003.

[7] Daniels, D.J., Ground Penetrating Radar, IET, 2004.

[8] Lombardi, F., Griffiths, H.D., and Balleri, A. Dependence of Landmine Radar Signature on Aspect Angle, IET Radar, Sonar \& Navigation, In press, doi: 10.1049/iet-rsn.2016.0491

[9] Giannakis, I., Xu, S., Aubry, P., Yarovoy, A., Sala, J. Signal processing for landmine detection using ground penetrating radar. IEEE International Geoscience and Remote Sensing Symposium (IGARSS), 2016, pp. 7442-7445.

[10] Daniels, D., Braunstein, J., and Nevard, M. Using minehound in Cambodia and Afghanistan. Journal of Conventional Weapons Destruction, 18, 2, p. 14, 2005.

[11] Sato, M., Fujiwara, J., Kido, T., and Takahashi, K. ALIS evaluation tests in Croatia, SPIE - Defense, Security, and Sensing, pp. 73031B-73031B, 2009.

[12] Lombardi, F., Griffiths, H.D., and Balleri, A. Influence of internal structure on landmine radar signatures, IEEE European Radar Conference 2016, pp. 161-164, 2016.

[13] Sai, B., and Ligthart, L.P., GPR phase-based techniques for profiling rough surfaces and detecting small, low-contrast landmines under flat ground, IEEE Transactions on Geoscience and Remote Sensing, 42, 2, pp. 318-326, 2004.

[14] Metwaly, M. Detection of metallic and plastic landmines using the GPR and 2-D resistivity techniques, Natural Hazards and Earth System Science, 7, 6, pp. 755-763, 2007.

[15] Lualdi, M., Lombardi, F. Combining orthogonal polarization for elongated target detection with GPR. Journal of Geophysics and Engineering, 11(5), p. 055006, 2014.

[16] Lualdi, M., Lombardi, F. Effects of antenna orientation on 3-D ground penetrating radar surveys: an archaeological perspective. Geophysical Journal International, 196(2), pp. 818-827, 2014.

[17] Lualdi, M., Lombardi, F. Significance of GPR polarisation for improving target detection and characterisation. Nondestructive Testing and Evaluation, 29(4), pp. 345-356, 2014.

[18] Habib, M.K. Humanitarian demining mine detection and sensors. IEEE International Symposium on Industrial Electronics, pp. 2237-2242, 2011.

[19] Daniels, D. J. A review of GPR for landmine detection. Sensing and Imaging: An international journal, 7(3), pp. 90-123, 2006.

[20] Lombardi, F., Griffiths, H. D., and Lualdi, M., The influence of spatial sampling in GPR surveys for the detection of landmines and IEDs. IEEE European Radar Conference 2016, pp. 322-325), 2016.

[21] Forte, E., Pipan, M. Review of multi-offset GPR applications: Data acquisition, processing and analysis, Signal Processing, 132, pp. 210220, 2016.

[22] Tarantola, A. Inverse problem theory and methods for model parameter estimation, SIAM, 2005

[23] Stiles, J. M., Parra-Bocaranda, P., and Apte, A., Detection of object symmetry using bistatic and polarimetric GPR observations. SPIE - Detection and Remediation Technologies for Mines and Minelike Targets, pp. 992-1002, 1999.

[24] Hamann, G., Tronicke, J., Steelman, C. M., Endres, A. L. Spectral velocity analysis for the determination of ground-wave velocities and their uncertainties in multi-offset GPR data. Near Surface Geophysics, 11(2), pp. 167-176, 2013.

[25] Zhao, W., Tian, G., Forte, E., Pipan, M., Wang, Y., Li, X., Shi, Z., Liu, H., Advances in GPR data acquisition and analysis for archaeology,Geophysical Journal International, 202(1), pp. 62-71, 2015.

[26] Counts, T., Gurbuz, A. C., Scott, W. R., McClellan, J. H., Kim, K. Multistatic ground-penetrating radar experiments. IEEE transactions on geoscience and remote sensing, 45(8), pp. 2544-2553, 2007.

[27] Sato, M., Bistatic GPR system for landmine detection using optical electric field, IEEE Antennas and Propagation Society International Symposium (Columbus, USA), 2, pp. 207-210, 2003.

[28] Jin, T., Lou, J., and Zhou, Z., Extraction of landmine features using a forward-looking ground-penetrating radar with MIMO array. IEEE Transactions on Geoscience and Remote Sensing, 50, 10, pp. 4135-4144, 2012.

[29] Stolt, R., Weglein, A.B. Seismic imaging and inversion, Cambridge University Press, 2012.

[30] Lualdi, M. True 3D Acquisition using GPR over small areas A cost effective solution, Proceedings of the Symposium on Application of Geophysics to Engineering and Environmental Problems (Charleston, USA), pp. 541550, 2011. 
[31] Lualdi, M., Lombardi, F. Orthogonal polarization approach for three dimensional georadar surveys. NDT\&E International, 60, pp. 87-99, 2013.

[32] Diamanti, N., and Annan, A. P. Characterizing the energy distribution around GPR antennas, Journal of Applied Geophysics, 99, pp. 83-90, 2013.

[33] Soldovieri, F., Lopera, O., and Lambot, S. Combination of Advanced Inversion Techniques for an Accurate Target Localization via GPR for Demining Applications, IEEE Transactions on Geoscience and Remote Sensing, 49, 1, pp. 451-461, 2011.

[34] Lopera, O., Slob, E.C., Milisavljevic, N., and Lambot, S. Filtering Soil Surface and Antenna Effects From GPR Data to Enhance Landmine Detection, IEEE Transactions on Geoscience and Remote Sensing, 45, 3, pp. 707-717, 2007.

[35] Eide, E., Vland, P.A., and Sala, J. Ground-coupled antenna array for step-frequency GPR, Proceedings of the 15th International Conference on Ground Penetrating Radar, Brussels, 2014, pp. 756-761.

[36] Lopera, O., Milisavljevi, N., and Lambot, S. Clutter reduction in GPR measurements for detecting shallow buried landmines: a Colombian case study, Near Surface Geophysics, 5(1), pp. 57-64, 2007.

[37] Hines, M. J., Piers, A., Du, K., Gonzalez-Valdes, B., Martnez-Lorenzo, J. ., and Rappaport, C. M., Localization of anti-personnel landmines using multi-bistatic ground-coupled ground penetrating radar, IEEE Radio Science Meeting, pp. 241-241, 2014.

[38] International Mine Action Standards, http://www.mineactionstandards. org/.

[39] Habib, M.K. Humanitarian demining: Difficulties, needs and the prospect of technology, IEEE International Conference on Mechatronics and Automation, pp. 213-218, 2008.

[40] Lualdi, M., and Zanzi, L. Testing a safe acquisition procedure for an effective application of GPR to security operations. Proceedings of the Symposium on Application of Geophysics to Engineering and Environmental Problems (Atlanta, USA), pp. 613623, 2005.

[41] Collaborative ORDnance data repository, James Madison University, http://ordata.info/.

[42] Yilmaz, Ö., Seismic data analysis. SEG, 2001. 